\title{
One-Day Construction Of Multiplex Arrays to Harness Natural CRISPR-Cas Systems
}

\author{
Robert M. Cooper, Jeff Hasty
}

Supporting Information 

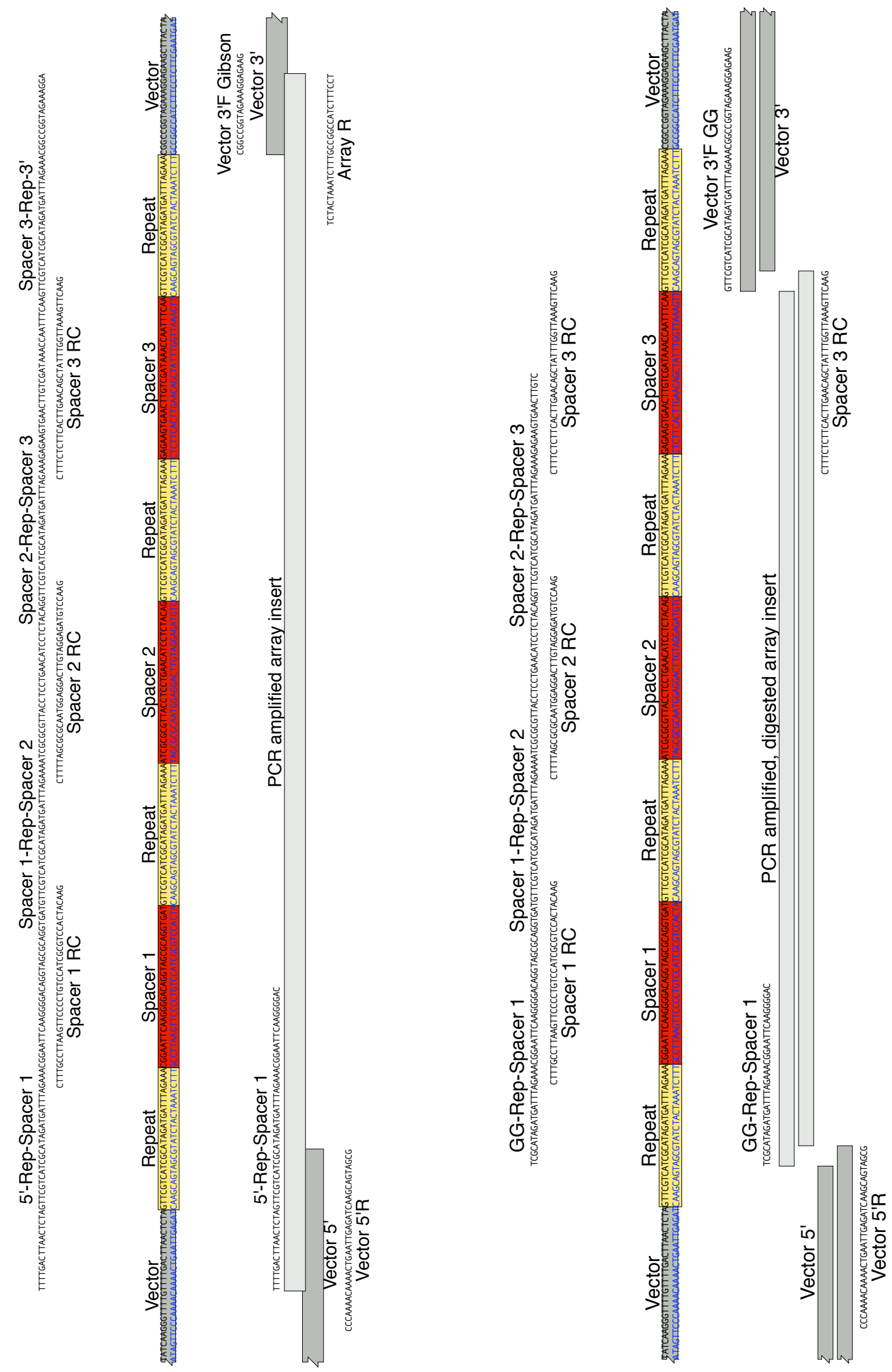

A

B 
Figure S1: Detailed multiplex natural CRISPR array assembly. A more detailed version of Figure 2 showing DNA sequences for the 3xBAP CRISPR array. The two array assembly strategies are for insertion into a vector using Gibson assembly or fusion PCR (A) or Golden Gate assembly (B). Note that primers used for Golden Gate assembly (denoted "GG" in B) have an additional BsaI site-containing tail appended to their $5^{\prime}$ ends that is not shown, specifically, TTTGGTCTCA. 

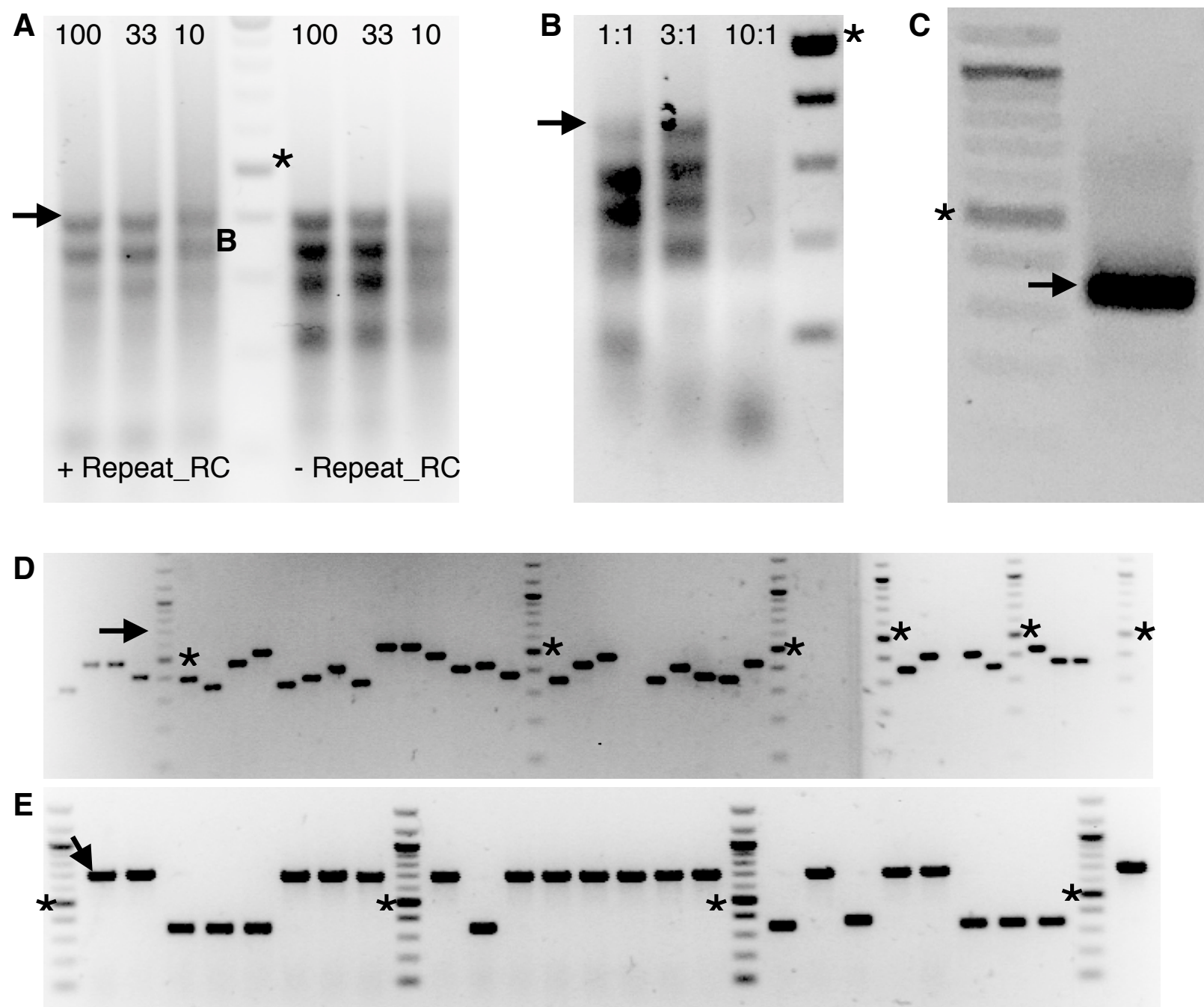

Figure S2: Multiplex array assembly optimization. Protocol optimizations were performed using a 6xIS-CRA array and inserted into pBAV using Golden Gate assembly. A,B: raw ligations, C: PCR amplification, D,E: Colony PCR screening of clones. Asterisks on all gels indicate the $500 \mathrm{bp}$ band of the ladder, and arrows indicate the correctly sized assembly. A) Including the Repeat_RC oligo increases incorrect, higher-molecular-weight smearing (left 3 vs right 3 lanes), and $100 \mathrm{uM}$ stock oligos (lanes 1 and 5) work better than $33 \mathrm{uM}$ (lanes 2 and 6) or $10 \mathrm{uM}$ (lanes 3 and 7) stock solutions. The center (lane 4) is a 100 bp ladder. B) Annealing and ligation is most efficient using 3 parts bottom oligos to 1 part top oligos. The lanes from left to right are ligations using 1:1, 3:1, and 10:1 ratios of bottom oligos to top oligos, followed by a 100 bp ladder. C) PCR amplification of the resulting ligation improves yield of the correctsized product. D) Golden Gate assembly directly from ligation products yielded no correct-sized arrays out of 36 tested clones. All of 6 sequenced clones were correct at the 3 ' end, but truncated at the 5' end of the array. E) As for D, but the ligation product was PCR amplified and gel extracted before inserting into the vector. 16 of 25 colonies were the correct size, and all incorrect clones had 0x arrays (a single repeat only). 

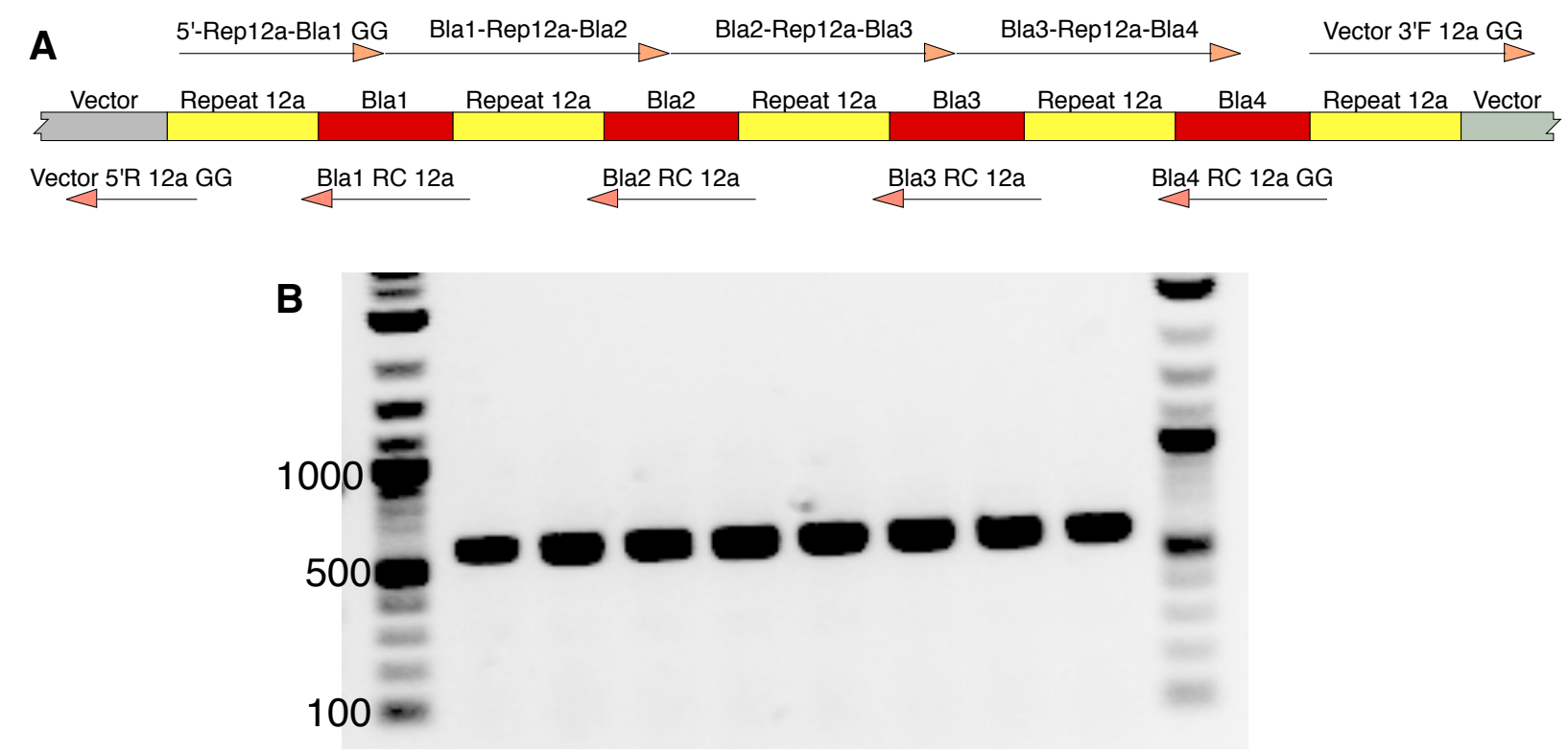

Figure S3: Assembly of a 4-spacer Cas12a array. A) Design and oligonucleotides for a 4spacer FnCas12a CRISPR array, to be inserted into the vector using Golden Gate assembly. This is analogous to Figure 2B for A. baylyi arrays. All oligos denoted by GG also contain a 5' Golden Gate tail (see Methods and Table S1). B) Screening of 8 clones for the 4-spacer array, of which all had the desired $603 \mathrm{bp}$ product. The primer pair hybridized to the backbone of the vector, outside the inserted CRISPR array. The ladder on the end lanes contains $100 \mathrm{bp}$ increments up to $1 \mathrm{~kb}$. 
Table S1: Oligos/Primers

\begin{tabular}{|c|c|c|}
\hline Purpose & Name & Sequence \\
\hline \multirow[t]{9}{*}{$\begin{array}{l}\text { CRISPR } 4 \text { 4Kan1 } \\
\text { Gibson }\end{array}$} & kan1 5'-R-T1 & $\begin{array}{l}\text { TTTTGACTTAACTCTAGTTCGTCATCGCATAGATGATTTAGAA } \\
\text { AGGTCGATCAGGGAGGA }\end{array}$ \\
\hline & kan1 T1-R-B1 & $\begin{array}{l}\text { TATCGGGGAAGAACAGGTTCGTCATCGCATAGATGATTTAGA } \\
\text { AATTGCATTCTAAAACCT }\end{array}$ \\
\hline & kan1 B1-R-T2 & $\begin{array}{l}\text { TAAATACAGAAAACAGGTTCGTCATCGCATAGATGATTTAGAA } \\
\text { AGTCGATACTATGTTAT }\end{array}$ \\
\hline & kan1 T2-R-B2 & $\begin{array}{l}\text { ACGCCAACTTTGAAAAGTTCGTCATCGCATAGATGATTTAGA } \\
\text { AAAAGCGAGCTCGGTACT }\end{array}$ \\
\hline & kan1 B2-R-3' & $\begin{array}{l}\text { AAAACAATTCATCCAGGTTCGTCATCGCATAGATGATTTAGAA } \\
\text { ACGGCCGGTAGAAAGGA }\end{array}$ \\
\hline & Kan1 T1 RC & GAACCTGTTCTTCCCCGATATCCTCCCTGATCGACCTTTC \\
\hline & Kan1 B1 RC & GAACCTGTTTTCTGTATTTAAGGTTTTAGAATGCAATTTC \\
\hline & Kan1 T2 RC & GAACTTTTCAAAGTTGGCGTATAACATAGTATCGACTTTC \\
\hline & Kan1 B2 RC & GAACCTGGATGAATTGTTTTAGTACCGAGCTCGCTTTTTC \\
\hline \multirow[t]{9}{*}{$\begin{array}{l}\text { CRISPR } 4 \times \text { ×an2 } \\
\text { Gibson }\end{array}$} & kan2 5'-R-T1 & $\begin{array}{l}\text { TTTTGACTTAACTCTAGTTCGTCATCGCATAGATGATTTAGAA } \\
\text { ATCGCCGTCGGGCATGC }\end{array}$ \\
\hline & kan2 T1-R-B1 & $\begin{array}{l}\text { GCGCCTTGAGCCTGGCGTTCGTCATCGCATAGATGATTTAGA } \\
\text { AAGGCTACCTGCCCATTC }\end{array}$ \\
\hline & kan2 B1-R-T2 & $\begin{array}{l}\text { GACCACCAAGCGAAACGTTCGTCATCGCATAGATGATTTAGA } \\
\text { AACAACCTTACCAGAGGG }\end{array}$ \\
\hline & kan2 T2-R-B2 & $\begin{array}{l}\text { CGCCCCAGCTGGCAATGTTCGTCATCGCATAGATGATTTAGA } \\
\text { AAGGCCGCTTGGGTGGAG }\end{array}$ \\
\hline & kan2 B2-R-3' & $\begin{array}{l}\text { AGGCTATTCGGCTATGGTTCGTCATCGCATAGATGATTTAGAA } \\
\text { ACGGCCGGTAGAAAGGA }\end{array}$ \\
\hline & Kan2 T1 RC & GAACGCCAGGCTCAAGGCGCGCATGCCCGACGGCGATTTC \\
\hline & Kan2 B1 RC & GAACGTTTCGCTTGGTGGTCGAATGGGCAGGTAGCCTTTC \\
\hline & Kan2 T2 RC & GAACATTGCCAGCTGGGGCGCCCTCTGGTAAGGTTGTTTC \\
\hline & Kan2 B2 RC & GAACCATAGCCGAATAGCCTCTCCACCCAAGCGGCCTTTC \\
\hline \multirow[t]{2}{*}{ Array PCR } & Array $\mathrm{R}$ & TCCTTTCTACCGGCCGTTTCTAAATCATCT \\
\hline & Array R GG & TTTGGTCTCATCCTTTCTACCGGCCGTTTCTAAATCATCT \\
\hline $\begin{array}{l}\text { CRISPR } 8 \times K a n \\
\text { Gibson }\end{array}$ & $\begin{array}{l}\text { Kan1 B2-R- } \\
\text { Kan2 T1 }\end{array}$ & $\begin{array}{l}\text { AAAACAATTCATCCAGGTTCGTCATCGCATAGATGATTTAGAA } \\
\text { ATCGCCGTCGGGCATGC }\end{array}$ \\
\hline Vector Gibson & Vector 5'R & TAGAGTTAAGTCAAAACAAAACCC \\
\hline
\end{tabular}




\begin{tabular}{|c|c|c|}
\hline & Vector 3'F & GAAACGGCCGGTAGAAAGGA \\
\hline \multirow[t]{2}{*}{$\begin{array}{l}\text { Vector Golden } \\
\text { Gate (GG) }\end{array}$} & Vector 5'R GG & $\begin{array}{l}\text { TTTGGTCTCAGCGATGACGAACTAGAGTTAAGTCAAAACAAA } \\
\text { ACCC }\end{array}$ \\
\hline & Vector 3'F GG & $\begin{array}{l}\text { TTTGGTCTCAGTTCGTCATCGCATAGATGATTTAGAAACGGC } \\
\text { CGGTAGAAAGGAGAAG }\end{array}$ \\
\hline \multirow{2}{*}{$\begin{array}{l}\text { Genomic } \\
\text { integrating } \\
\text { CRISPR vector }\end{array}$} & pp 5’F & TGAGCCGACATTTTATTACCCTCT \\
\hline & pp 3’R & TTACCTGAAAGCCAATCGCTG \\
\hline \multirow[t]{6}{*}{$\mathrm{CRISPR}_{3 \times \mathrm{BAP}} \mathrm{GG}$} & GG-R-BAP1 & $\begin{array}{l}\text { TTTGGTCTCATCGCATAGATGATTTAGAAACGGAATTCAAGG } \\
\text { GGAC }\end{array}$ \\
\hline & BAP1-R-BAP2 & $\begin{array}{l}\text { AGGTAGCGCAGGTGATGTTCGTCATCGCATAGATGATTTAGA } \\
\text { AAATCGCGCGTTACCTCC }\end{array}$ \\
\hline & BAP2-R-BAP3 & $\begin{array}{l}\text { TGAACATCCTCTACAGGTTCGTCATCGCATAGATGATTTAGAA } \\
\text { AGAGAAGTGAACTTGTC }\end{array}$ \\
\hline & BAP1 RC & GAACATCACCTGCGCTACCTGTCCCCTTGAATTCCGTTTC \\
\hline & BAP2 RC & GAACCTGTAGAGGATGTTCAGGAGGTAACGCGCGATTTTC \\
\hline & BAP3 RC GG & $\begin{array}{l}\text { TTTGGTCTCAGAACTTGAAATTGGTTTATCGACAAGTTCACTT } \\
\text { CTCTTTC }\end{array}$ \\
\hline \multirow[t]{7}{*}{$\begin{array}{l}\text { CRISPR } 3 \times \text { CRA-3×BAP } \\
\text { GG }\end{array}$} & GG-R-CRA1 & $\begin{array}{l}\text { TTTGGTCTCATCGCATAGATGATTTAGAAATCTCCGCGCTTG } \\
\text { CTTC }\end{array}$ \\
\hline & CRA1-R-CRA2 & $\begin{array}{l}\text { GCATAATGCAGATTGAGTTCGTCATCGCATAGATGATTTAGAA } \\
\text { AGTCACTATGACCATGT }\end{array}$ \\
\hline & CRA2-R-CRA3 & $\begin{array}{l}\text { TGCTTTGTATTGTGAAGTTCGTCATCGCATAGATGATTTAGAA } \\
\text { ACCCGGATTTTGACTGG }\end{array}$ \\
\hline & CRA3-R-BAP1 & $\begin{array}{l}\text { CGAAATGTAGAAGATAGTTCGTCATCGCATAGATGATTTAGAA } \\
\text { ACGGAATTCAAGGGGAC }\end{array}$ \\
\hline & CRA1 RC & GAACTCAATCTGCATTATGCGAAGCAAGCGCGGAGATTTC \\
\hline & CRA2 RC & GAACTTCACAATACAAAGCAACATGGTCATAGTGACTTTC \\
\hline & CRA3 RC & GAACTATCTTCTACATTTCGCCAGTCAAAATCCGGGTTTC \\
\hline \multirow{2}{*}{$\begin{array}{l}\text { PCR screening of } \\
\text { arrays }\end{array}$} & Array screen F & GGAGTTCTGAGGTCATTACTGGATCTA \\
\hline & Array screen $\mathrm{R}$ & CAAATGTACGGCCAGCAACG \\
\hline \multirow{2}{*}{$\begin{array}{l}\text { bap deletion } \\
\text { donor DNA }\end{array}$} & BAP 5'F & AGCAGCTGAGAGCCTGAATG \\
\hline & BAP 5'R & ACATGCCAGCACTTAATCTGA \\
\hline
\end{tabular}




\begin{tabular}{|c|c|c|}
\hline & BAP 3'F & $\begin{array}{l}\text { TCAGATTAAGTGCTGGCATGTGCACCCAATCCCTAACATTAA } \\
\text { ACA }\end{array}$ \\
\hline & BAP 3'R & GGTTCGGGCACCTCATCATT \\
\hline \multirow{4}{*}{$\begin{array}{l}\text { CRAФ deletion } \\
\text { donor DNA }\end{array}$} & CRA 5'F & ACAGGGCAGCCATTAACTGA \\
\hline & CRA 5'R & TCTGAGACTGTAGCCTACGCA \\
\hline & CRA 3'F & $\begin{array}{l}\text { TGCGTAGGCTACAGTCTCAGAACGAAGTTATGTGCCACAAG } \\
\text { AAA }\end{array}$ \\
\hline & CRA 3'R & TCAGACGCAAGCGTGAAGAT \\
\hline \multirow{2}{*}{$\begin{array}{l}\text { bap deletion } \\
\text { screening }\end{array}$} & BAP checkF & GCCTCCTAAAATTGGGGGCT \\
\hline & BAP checkR & CTTGGTTCTGCATTGGGTGC \\
\hline \multirow{4}{*}{$\begin{array}{l}\text { CRAФ deletion } \\
\text { screening }\end{array}$} & CRA checkF & GACTTGCGTAGGCTTGGACT \\
\hline & CRA checkR & GCATGTCATGGTTTGGTGGG \\
\hline & CRA circular F & ATGAACGCGATCATTGCAGC \\
\hline & CRA circular $\mathrm{R}$ & TACGGCCAATTGATCACCCA \\
\hline \multirow[t]{10}{*}{$\begin{array}{l}\text { Cas } 12 a / C p f 1 \\
\text { CRISPR }_{4 \times B l a} \text { array }\end{array}$} & GG-R12a-Bla1 & $\begin{array}{l}\text { TTTGGTCTCATAAGAACTTTAAATAATTTCTACTGTTGTAGATC } \\
\text { GGCGTCAATACGGGA }\end{array}$ \\
\hline & Bla1-R12a-Bla2 & $\begin{array}{l}\text { TAATACCGCGCCACATGTCTAAGAACTTTAAATAATTTCTACT } \\
\text { GTTGTAGATGGAGCTGAATGAAGCC }\end{array}$ \\
\hline & Bla2-R12a-Bla3 & $\begin{array}{l}\text { ATACCAAACGACGAGCGTCTAAGAACTTTAAATAATTTCTACT } \\
\text { GTTGTAGATCTCCCGTATCGTAGTT }\end{array}$ \\
\hline & Bla3-R12a-Bla4 & $\begin{array}{l}\text { ATCTACACGACGGGGAGTCTAAGAACTTTAAATAATTTCTACT } \\
\text { GTTGTAGATAGCCGGAAGGGCCGAG }\end{array}$ \\
\hline & $\begin{array}{l}\text { Vector 3'F 12a } \\
\text { GG }\end{array}$ & $\begin{array}{l}\text { TTTGGTCTCAGTCTAAGAACTTTAAATAATTTCTACTGTTGTA } \\
\text { GATCGGCCGGTAGAAAGGACA }\end{array}$ \\
\hline & $\begin{array}{l}\text { Vector 5'R } 12 \mathrm{a} \\
\text { GG }\end{array}$ & TTTGGTCTCACTTAGACTAGAGTTAAGTCAAAACAAAACCC \\
\hline & Bla1 RC 12a & AGACATGTGGCGCGGTATTATCCCGTATTGACGCCGATCT \\
\hline & Bla2 RC 12a & AGACGCTCGTCGTTTGGTATGGCTTCATTCAGCTCCATCT \\
\hline & Bla3 RC 12a & AGACTCCCCGTCGTGTAGATAACTACGATACGGGAGATCT \\
\hline & Bla4 RC 12a & $\begin{array}{l}\text { TTTGGTCTCAAGACCAGGACCACTTCTGCGCTCGGCCCTTC } \\
\text { CGGCTATCT }\end{array}$ \\
\hline
\end{tabular}


Table S2: CRISPR spacers

\begin{tabular}{l|l}
\hline Name & Sequence \\
\hline Kan1_T1 & GGTCGATCAGGGAGGATATCGGGGAAGAACAG \\
\hline Kan1_T2 & GTCGATACTATGTTATACGCCAACTTTGAAAA \\
\hline Kan1_B1 & TTGCATTCTAAAACCTTAAATACAGAAAACAG \\
\hline Kan1_B2 & AAGCGAGCTCGGTACTAAAACAATTCATCCAG \\
\hline Kan2_T1 & TCGCCGTCGGGCATGCGCGCCTTGAGCCTGGC \\
\hline Kan2_T2 & CAACCTTACCAGAGGGCGCCCCAGCTGGCAAT \\
\hline Kan2_B1 & GGCTACCTGCCCATTCGACCACCAAGCGAAAC \\
\hline Kan2_B2 & GGCCGCTTGGGTGGAGAGGCTATTCGGCTATG \\
\hline CRA1 & TCTCCGCGCTTGCTTCGCATAATGCAGATTGA \\
\hline CRA2 & GTCACTATGACCATGTTGCTTTGTATTGTGAA \\
\hline CRA3 & CCCGGATTTTGACTGGCGAAATGTAGAAGATA \\
\hline BAP1 & CGGAATTCAAGGGGACAGGTAGCGCAGGTGAT \\
\hline BAP2 & ATCGCGCGTTACCTCCTGAACATCCTCTACAG \\
\hline BAP3 & GAGAAGTGAACTTGTCGATAAACCAATTTCAA \\
\hline Random & TAGGGGAAAGCCTACTAGCCGGAGTGTTGCGA \\
\hline
\end{tabular}

DNA sequence of sample genomically integrating vector, $p$ p2.1-CRISPR ${ }_{8 \times K a n}-S p e c-$ pp2.2

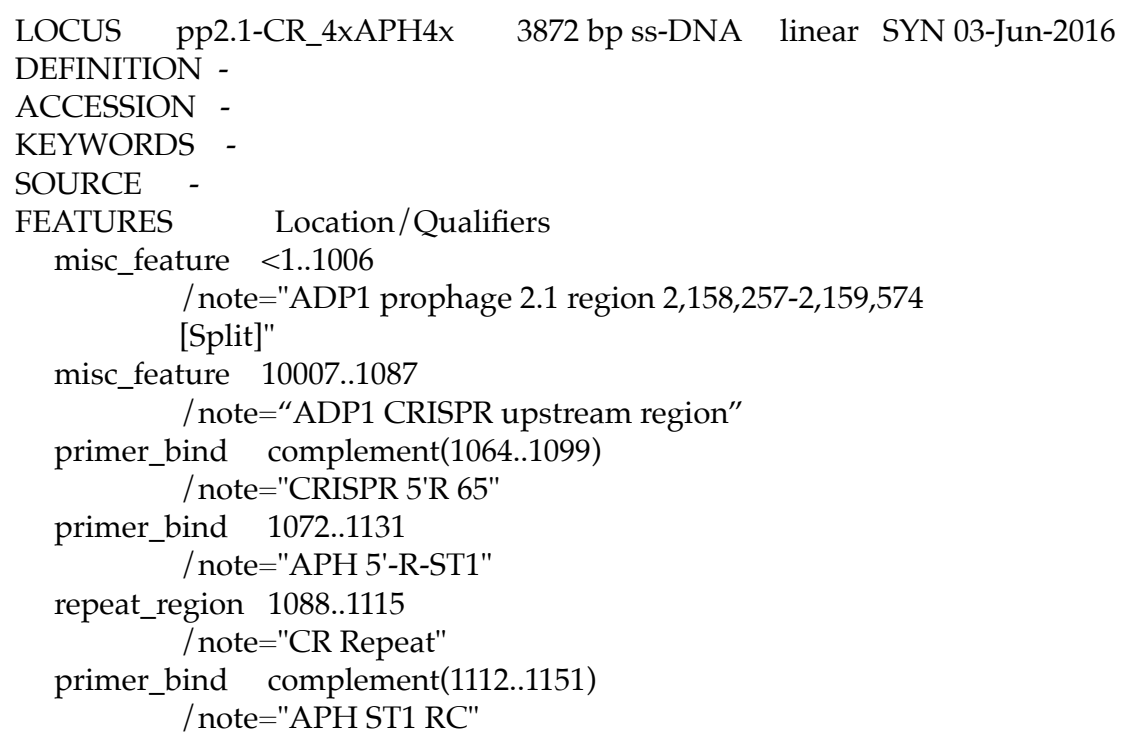




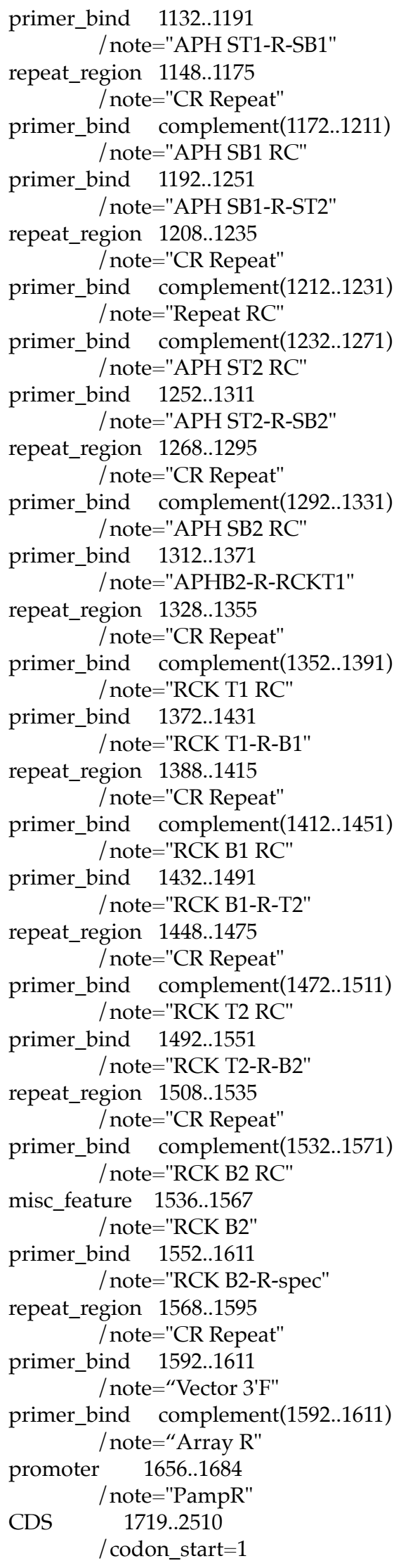


/db_xref="GI:336359759"

/gene="specR"

/ note="spectinomycin resistance marker"

/ product="SpecR"

/ protein_id="AEI53620.1"

/ transl_table $=11$

/ translation="MREAVIAEVSTQLSEVVGVIERHLEPTLLAVHLYGSAVDGGLKPH

SDIDLLVTVTVRLDETTRRALINDLLETSASPGESEILRAVEVTIVVHDDIIPWRYPAK

RELQFGEWQRNDILAGIFEPATIDIDLAILLTKAREHSVALVGPAAEELFDPVPEQDLF

EALNETLTLWNSPPDWAGDERNVVLTLSRIWYSAVTGKIAPKDVAADWAMERLPAQYQP

VILEARQAYLGQEEDRLASRADQLEEFVHYVKGEITKVVGK"

misc_feature 2848..3872

/ note="ADP1 prophage 2.2 region 2,161,721-2,162,745"

primer_bind complement(3852..3872)

/ note="pp2.2 R63"

BASE COUNT $1003 \mathrm{~A} \quad 873 \mathrm{C} \quad 886 \mathrm{G} \quad 1110 \mathrm{~T} \quad$ 0 OTHER

ORIGIN ?

1 TGAGCCGACA TTTTATTACC CTCTTATCAA ACCGTACCTT TCACATAACG AATGAATGAA 61 TACCGTACAT GGAGTGCGGC CAACCCACAG CGAACATCAT ATTTCGCATC CATCACCGTA 121 CGGTTTTCCG TTTTAAGCTC TGCCCATGAT CTATCATGGA AATAACGGCT AATGATCACC 181 TGCATCCACT CAAGTGTCGT TTCACTGTCT GTACCATTAA TAATATCCAG TACTAAACGT 241 TGTACGGCAC GAGCTTCATT ATCGTTAATC TGACACGACA CTTTGTGACG TATAGCTTGT 301 TGTACTCCTT GAGCATCACA AAGGTAATAA GCAATAAGTT TAGCTCGATC TTTCTTCTTT 361 ACACGTACCT TGGCTTTCTT CATTGCAATA GCAATCGGGC TATCGGAATA CTGTCCACCA 421 CGACAAGAAC GTTGCCACGC TCCACATTGG CGTAACCAAT CTGGCAAATC ATACTTGCTC 481 CAATCCACCG TCTGCATAAT GTGCACTGCT GTATTCATCT CATCACCTAA TTTGTTTCAA 541 GTTAAATTTT ATAAGCGTTA TTGTTTTATG GTTCTGCCTG CTCCTCTACC GATCTAAAAC 601 GACAAGTTTC GAGATAATCC AGTACTCGAA CTGCACCGCG TTTACCGTGT CGGTTTTTCA 661 CTACAATCAG CTCTGTGATT CCCATCGGTT TGGTTGAGTC TTCTGGATCG GTTAGGGGGT 721 TAACAAGGAT GATCTGGTCT GCATCTTGCT CGATTTGTCC AGATTCTTTG ATATCTGATG 781 CTTTAGGACG TTTGCCCTTC TCTGCCTCAC GGTTGAGCTG TACCAGTGCA ATGACAGGAC 841 ATTCAAACTC TTTCGCCATG GATTTTAATT CACGGCTGAT GGAACTGACT TCCTGAAAGC 901 GATCTTTCTT GCTCGGGTCT CTGAGCAGTT GTAAATAATC CACGATGATG CAGCCCAATT 961 CCTTGTAACG GCGTTTGGCT CGACGTGCAT AGGAACGGAC CTCACTCAAG TGATTCATAA 1021 CGAAGTATTT TTACTCATTA AAAGCTTATA TAATTGATAT CAAGGGTTTT GTTTTGACTT 1081 AACTCTAGTT CGTCATCGCA TAGATGATTT AGAAAGGTCG ATCAGGGAGG ATATCGGGGA 1141 AGAACAGGTT CGTCATCGCA TAGATGATTT AGAAATTGCA TTCTAAAACC TTAAATACAG 1201 AAAACAGGTT CGTCATCGCA TAGATGATTT AGAAAGTCGA TACTATGTTA TACGCCAACT 1261 TTGAAAAGTT CGTCATCGCA TAGATGATTT AGAAAAAGCG AGCTCGGTAC TAAAACAATT 1321 CATCCAGGTT CGTCATCGCA TAGATGATTT AGAAATCGCC GTCGGGCATG CGCGCCTTGA 1381 GCCTGGCGTT CGTCATCGCA TAGATGATTT AGAAAGGCTA CCTGCCCATT CGACCACCAA 1441 GCGAAACGTT CGTCATCGCA TAGATGATTT AGAAACAACC TTACCAGAGG GCGCCCCAGC 1501 TGGCAATGTT CGTCATCGCA TAGATGATTT AGAAAGGCCG CTTGGGTGGA GAGGCTATTC 1561 GGCTATGGTT CGTCATCGCA TAGATGATTT AGAAACGGCC GGTAGAAAGG AGAAGCTTAC 1621 TAGCTATTTG TTTATTTTTC TAAATACATT CAAATATGTA TCCGCTCATG AGACAATAAC 1681 CCTGATAAAT GCTTCAATAA TATTGAAAAA GGAAGAGTAT GAGGGAAGCG GTGATCGCCG 1741 AAGTATCGAC TCAACTATCA GAGGTAGTTG GCGTCATCGA GCGCCATCTC GAACCGACGT 1801 TGCTGGCCGT ACATTTGTAC GGCTCCGCAG TGGATGGCGG CCTGAAGCCA CACAGTGATA 1861 TTGATTTGCT GGTTACGGTG ACCGTAAGGC TTGATGAAAC AACGCGGCGA GCTTTGATCA 1921 ACGACCTTTT GGAAACTTCG GCTTCCCCTG GAGAGAGCGA GATTCTCCGC GCTGTAGAAG 1981 TCACCATTGT TGTGCACGAC GACATCATTC CGTGGCGTTA TCCAGCTAAG CGCGAACTGC 2041 AATTTGGAGA ATGGCAGCGC AATGACATTC TTGCAGGTAT CTTCGAGCCA GCCACGATCG 2101 ACATTGATCT GGCTATCTTG CTGACAAAAG CAAGAGAACA TAGCGTTGCC TTGGTAGGTC 2161 CAGCGGCGGA GGAACTCTTT GATCCGGTTC CTGAACAGGA TCTATTTGAG GCGCTAAATG 2221 AAACCTTAAC GCTATGGAAC TCGCCGCCCG ACTGGGCTGG CGATGAGCGA AATGTAGTGC 2281 TTACGTTGTC CCGCATTTGG TACAGCGCAG TAACCGGCAA AATCGCGCCG AAGGATGTCG 2341 CTGCCGACTG GGCAATGGAG CGCCTGCCGG CCCAGTATCA GCCCGTCATA CTTGAAGCTA 2401 GACAGGCTTA TCTTGGACAA GAAGAAGATC GCTTGGCCTC GCGCGCAGAT CAGTTGGAAG 


\begin{abstract}
2461 AATTTGTCCA CTACGTGAAA GGCGAGATCA CCAAGGTAGT CGGCAAATAA TGTCTAACAA 2521 TTCGTTCAAG CCGAGGGGCC GCAAGATCCG GCCACGATGA CCCGGTCGTC GGTTCAGGGC 2581 AGGGTCGTTA AATAGCCGCT TATGTCTATT GCTGGTTTAC CGGTTTATTG ACTACCGGAA 2641 GCAGTGTGAC CGTGTGCTTC TCAAATGCCT GAGGTTTCAG CAAAAAACCC CTCAAGACCC 2701 GTTTAGAGGC CCCAAGGGGT TATGCTAGTT ATTGCTCAGC GGTGGCAGCA GCCTAGGTTA 2761 ATTAAGCTGC GCTAGTAGAC GAGTCCATGT GCTGGCGTTC AAATTTCGCA GCAGCGGTTT 2821 CTTTACCAGA CTCGACAAGC TTACTAGAGT GTTCATATTG ACCTCGCTTA GTGTGGTTAA 2881 TACGCCGCTT CTTGTTACTG CAAGAGGCGG TTTTTTTATG GGTGTACACA TGACTGCACC 2941 TGTTGATTCA GTTCAGATAG TGCTTTGTGC ATCTCATGGA TGACTCTGGC CATATCCAGT 3001 GCTTCGCCTT GGGTAATTCG CCCATCGGCC ATCATTTCTT TAAACAGTGC TGATATATCG 3061 CCCTTCTTGA TGCCTATGCA TAGGAAGGTA TCCATCAGAC TGGTATCTCG CTGGCTCTCG 3121 GGTATGTCTG GCAGGTCAAT TGCCACCTTT CCGAGTCGTG CACACATTTC CTGCAATATC 3181 CGATAGTCCC CTGTAATCTC CATCAGCTTG ACTGCCTCGA GCAATGTAAT GTGATGGGTA 3241 TGTGTGTTTG GGTTGACCTT GCTATTGAGC ACCGCAGGGC TTTTGATGCC TAAACGTGAT 3301 GCAAGTGCAG ATGCACCACC CAGAAAGTCG TGAACGGTGT GATAGGCAGC ATCTAATATG 3361 TTCATGGCGG GTTCCTTTGA ACGTGTTTAT TAGATGGGTG CTGACATAAG ATTGGATTTA 3421 TGGTTAGGAC GTAATTCAAT CCAAATATCT TCGTAATCAT CTGGAAATAA ATCTTTGCGA 3481 GTGCATAGAC CTTTATCTTC AGCAATTACA GCTAAGCGGA TTTTGCGATC TCTGGGAATT 3541 GCTTTCCATC CACTTACGGA TGCAGCGGTG ATACCTAAAA ATCTAGCAAC AGCAGTTACA 3601 CCACCTAAAA GCTCAATAAA TTGATCATCA GTCATGTTGA TCTCCTAATT TTATTGCCTC 3661 AATTATTAGG TATTCCTTAT ATTTTATCAA TAGGAATACC TTATTTATTT TATGTTAGGA 3721 TTTCCTAATA GACTAGGTAA GATCATGAAA ACATTAGCTG AACGACTTAA ATATGCGATG 3781 GAAATTTTGC CACCTAAGAA AATCAAGGGT GTCGAACTTG CTCGTGTAGT TGGAGTTAAA 3841 CCACCATCTG TCAGCGATTG GCTTTCAGGT AA
\end{abstract} //

\title{
DNA sequence of sample replicating vector, $\mathrm{pBAV1spec-CRISPR_{3 \times CRA } - S p e c}$
}

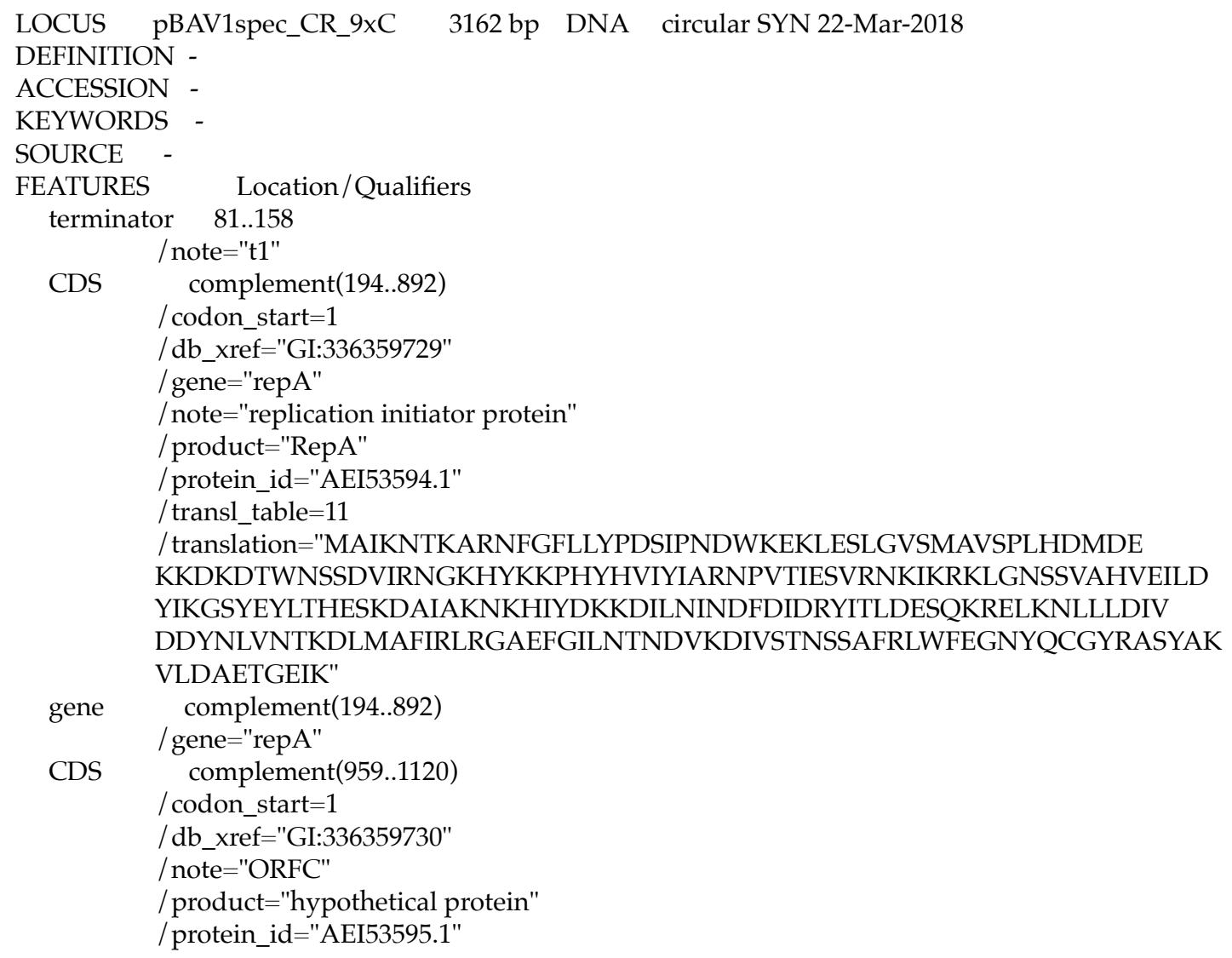




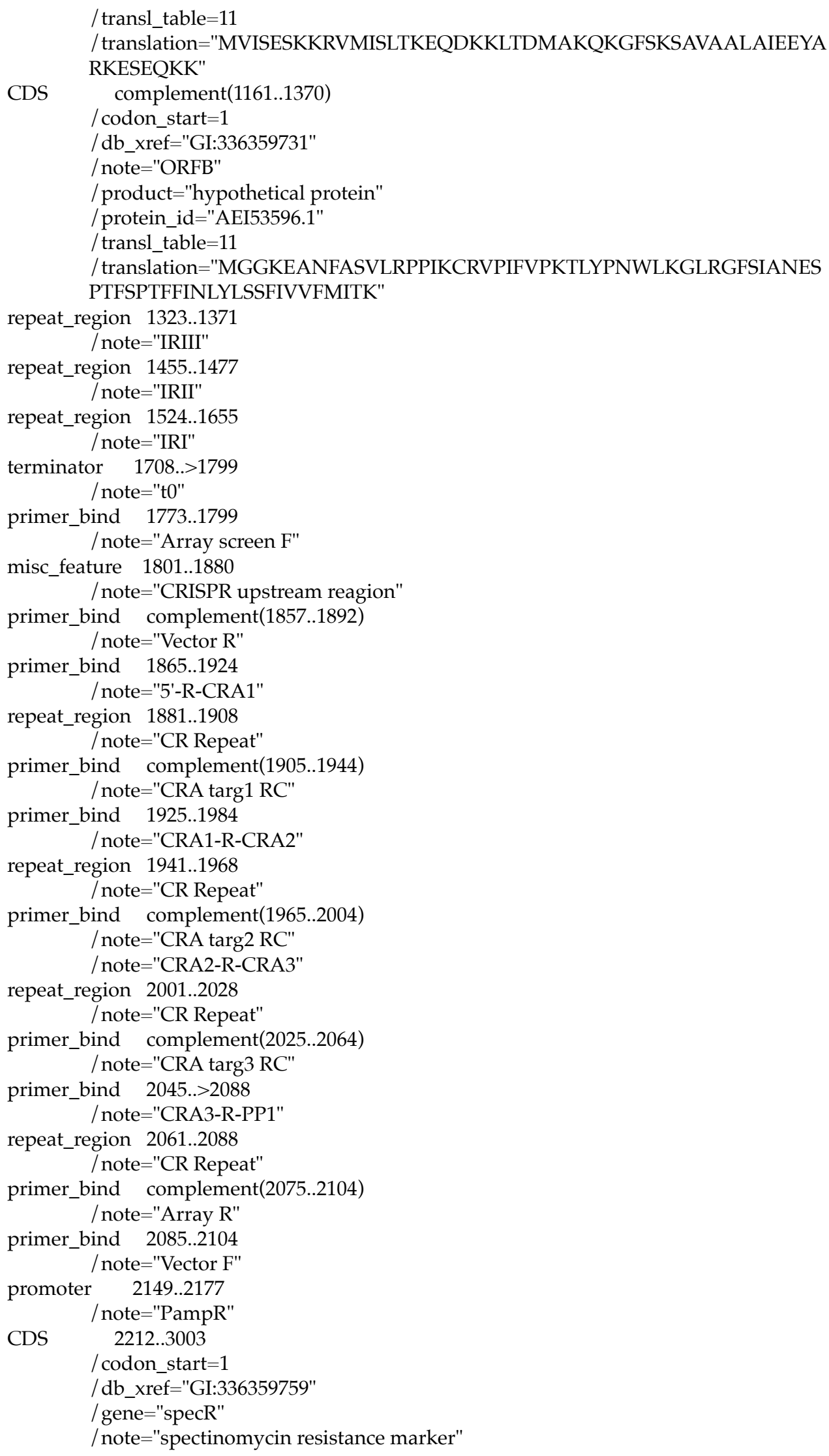




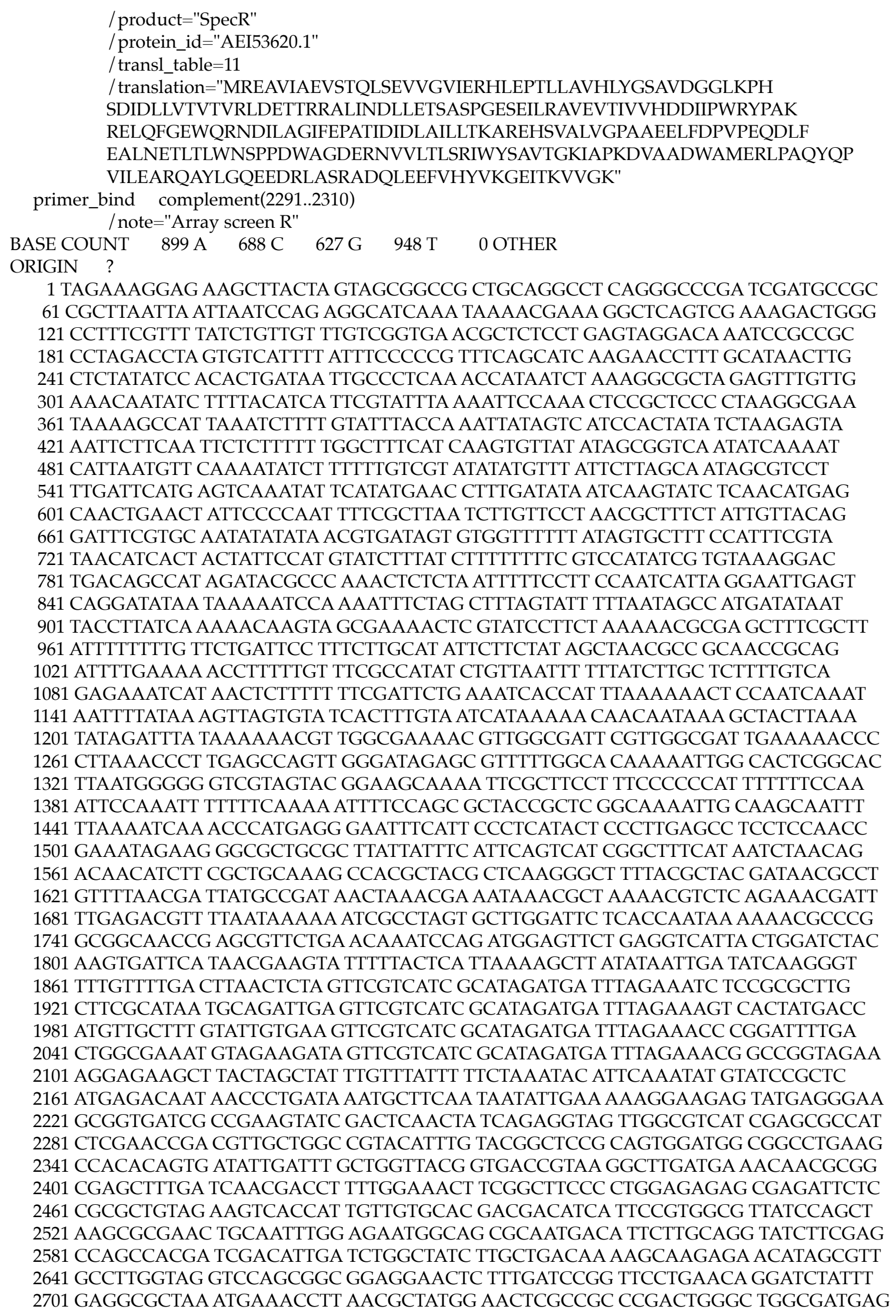


2761 CGAAATGTAG TGCTTACGTT GTCCCGCATT TGGTACAGCG CAGTAACCGG CAAAATCGCG 2821 CCGAAGGATG TCGCTGCCGA CTGGGCAATG GAGCGCCTGC CGGCCCAGTA TCAGCCCGTC 2881 ATACTTGAAG CTAGACAGGC TTATCTTGGA CAAGAAGAAG ATCGCTTGGC CTCGCGCGCA 2941 GATCAGTTGG AAGAATTTGT CCACTACGTG AAAGGCGAGA TCACCAAGGT AGTCGGCAAA 3001 TAATGTCTAA CAATTCGTTC AAGCCGAGGG GCCGCAAGAT CCGGCCACGA TGACCCGGTC 3061 GTCGGTTCAG GGCAGGGTCG TTAAATAGCC GCTTATGTCT ATTGCTGGTT TACCGGTTTA 3121 TTGACTACCG GAAGCAGTGT GACCGTGTGC TTCTCAAATG CC // 